\title{
Marketing y las nuevas tecnologías de información, aplicadas en los negocios ${ }^{1}$. Marketing and new information technologies, applied in business.
}

\author{
Ricardo Andrade Díaz
}

Artículo recibido en octubre de 2014; artículo aceptado en enero de 2015

Citación del artículo: Andrade, R. (2014). Marketing y las nuevas tecnologías de información, aplicadas en los negocios. I+D Revista de Investigaciones, 4(2),34-48.

\section{Resumen}

Las Sociedades de comercialización internacional (C.I) de Cúcuta, tienen como propósito fomentar la exportación regional. Sin embargo, las empresas mantienen la tradición de vender preferiblemente al mercado de Venezuela, el cual se encuentra en una profunda crisis económica, política y social, afectando el flujo comercial binacional; que promueve la actividad económica informal, por lo cual, es imperiosa la necesidad de identificar nuevos segmentos de mercado en países distintos al mencionado. El objetivo del estudio es proponer la implementación de estrategias de E-marketing y E-commerce en el sector comercio exterior. La investigación diseñada tiene un enfoque positivista, de tipo descriptivo; se abordó una muestra de 126 C.I, mediante el método muestreo probabilístico, aleatorio simple para población finita, con un margen de error de 4\% y un nivel de confianza de 94\%. Se aplicó un formulario compuesto por 45 preguntas y una entrevista estructurada; a partir del diseño de perfiles según la variable estudiada (Gerencia, producto, plaza, precio, promoción y tecnología). Por lo anterior, ¿Cuál sería el impacto en las ventas de las C.I de Cúcuta mediante la implementación de estrategias de mercadeo digital en el mercado global?

Palabras clave: Mercadeo digital, Comercio electrónico, Marketing, Comercio internacional, Tecnologías de información.

\footnotetext{
${ }^{1}$ Artículo de tipo estudio empírico, tipo de enfoque (cuantitativo), resultado de un proyecto de investigación culminado), perteneciente al área de gerencia de empresas, sub área de mercadeo, desarrollado en el grupo de investigación Confines y fue financiado por la Universidad Nacional Experimental del Táchira - UNET de la ciudad de San Cristóbal (Venezuela). Dirección Avenida Universidad, Sector Paramillo. San Cristóbal, Venezuela, PBX: +58 (276) 353-0422. Fecha de inicio: 15 de Febrero de 2009, fecha de terminación: 20 de Mayo de 2010.

${ }^{2}$ Administrador de Negocios, Universidad de San Buenaventura. Magister en Gerencia de Empresas mención Mercadeo, Universidad Nacional Experimental del Táchira. Docente- investigador del grupo: Confines Universidad de Santander UDES de la ciudad de Bucaramanga (Colombia): Dirección: Campus Universitario Lagos del Cacique Calle 70 No. 55-210, PBX:+57 (7) 6516500. Correo electrónico institucional: ri.andrade@mail.udes.edu.co
} 


\section{Abstract}

International marketing societies (CI) of Cucuta are intended to promote regional exportation. However, companies maintain the tradition of selling preferably to the Venezuelan market, which is in a deep economic, political and social crisis affecting binational trade flow; promoting informal economic activity, so it is imperative to identify new market segments in different countries other than the above mentioned. The aim of the study is to propose the implementation of digital marketing strategies in the external trade sector. The designed research, has a positivist approach, in a descriptive way; a sample of 126 (C.I) was addressed through the probability sampling method, simple random for finite population, with a margin of error of $4 \%$ and a confidence level of $94 \%$. A form composed by 45 questions was applied and a structured interview as well; According to the profiles design, depending on the variables (Management, product, place, price, promotion and technology). Therefore, what would be the impact on sales of the C.I's in Cucuta by implementing E-marketing and E-commerce strategies in the global market?

Keywords: Digital Marketing, E-Commerce, Marketing, International trade, Information and communication Technologies.

\section{Introducción}

Las empresas del municipio de Cúcuta, se caracterizan por la vocación tradicional a centrar su actividad económica en el sector comercio. Esta actividad está enfocada hacia el intercambio comercial con Venezuela, situación que se presenta debido a la posición geográfica de frontera, lo que permite realizar un intercambio comercial natural con el principal socio económico desde hace décadas. A través de la investigación, se observó y analizó la situación del intercambio binacional, por lo cual, cabe destacar que la acción de marketing, la cual "consiste en un proceso administrativo y social gracias al cual determinados grupos o individuos obtienen lo que necesitan o desean a través del intercambio de productos o servicios (Kotler y Armstrong, 2003), carece de un proceso de gestión organizado y formal, para dar cumplimiento a los objetivos de la organización (Stanton, Etzel y Walker 2007), las actividades de mercadeo usadas por las C.I se resume en el establecimiento de un canal de distribución elemental, orientado en prácticas tradicionales, ya que se fundamenta en la identificación de empresas en Venezuela y los empresarios toman la decisión de realizar viajes de negocio de manera física, lo cual evidencia varios factores que limitan su alcance como los altos gastos que implican el desarrollo de dichas actividades; los contactos con los compradores potenciales que se logra establecer, son deficientes en términos de amplitud e impacto, factores como el tiempo, dan como resultado estrategias poco efectivas y el incumplimiento de los objetivos propuestos.

En consecuencia, y debido a que el flujo comercial bilateral se encuentra constantemente afectado por las políticas comerciales implementadas por parte del gobierno de Venezuela, las cuales son sustentadas por discursos políticos alimentados con sentimientos socialistas y económicos unilaterales en complejas disputas y desacuerdos internos de 
este país, según el artículo de la revista de la $\mathrm{BBC}$, donde se hace referencia al efecto de las relaciones políticas y sociales sobre el impacto de las relaciones comerciales entre Colombia y Venezuela, que en consecuencia, ha generado en los empresarios, la imperiosa necesidad de identificar nuevos nichos de mercado, donde vender la producción regional y nacional, como lo ratifica portafolio.co; a través de la segmentación del mercado, definida como el procedimiento de dividir un mercado en distintos subconjuntos de consumidores que tienen necesidades o características comunes...... (Shiffman y Kanuk, 2005), y efectivas estrategias de comunicación de marketing, que incluye a la publicidad, pero también una serie de técnicas de comunicación relacionadas que se utilizan en marketing como promoción de ventas, relaciones públicas, respuesta directa, eventos y patrocinio, empaque y venta personal (Wells, Moriarty y Burnett, 2007), y el Emarketing, que tiene como objetivo la incorporación y uso de los sistemas y tecnologías de la información a la práctica del marketing (Martí, 2011).

Así mismo, la planificación de marketing, entendida como el proceso estructurado de investigación y análisis de la situación de marketing; el planteamiento y justificación de los objetivos de marketing, estrategias y programas; y la puesta en marcha, evaluación y control de las actividades necesarias para lograr los objetivos (Wood, 2004), se requiere entonces visualizar escenarios empresariales orientados hacia la nueva economía, la digitalización de información y en la respectiva infraestructura de las TIC (Zysman y Weber, 2000), mediante el diseño e implementación de modelos de negocio soportados en internet. Y en ese sentido "La economía digital es el nuevo entorno económico en el que predomina el uso de las tecnologías de la información y la comunicación para hacer transacciones de negocios entre los agentes económicos"(Duarte, 2010).

En ese sentido, la web 3.0 marca los principios para crear una base de conocimiento e información semántica y cualitativa. Se pretende con ello, almacenar las preferencias de los usuarios (Hernández y Küster, 2012).

En este orden de ideas, el comercio electrónico se refiere a los intercambios mediados por la tecnología entre diversas partes (individuos, organizaciones, o ambos), así como las actividades electrónicas dentro y entre organizaciones que facilitan esos intercambios (Rayport y Jaworsky, 2001), por lo cual es significativo explorar cómo los modelos de transacciones de compra y venta están cambiando, y cómo los ingresos resultan afectados, así el E-commerce es por tanto una forma de venta en la que no existe establecimiento físico ni contacto directo entre vendedor y comprador (Cruz, 2009), de esta manera se logrará diseñar adecuadas estrategias de mercadeo y acciones de E-marketing y publicidad interactiva, definida como toda acción publicitaria desarrollada en medios interactivos (Martí, 2011).

En tal sentido, determinar ¿Cuál sería el impacto en las ventas de las C.I de Cúcuta, mediante la implementación de estrategias de Emarketing? corresponde a la pregunta central de la investigación, la cual, a través de la elaboración de la propuesta de investigación, 
permitirá establecer escenarios de comunicación efectiva para las diferentes estrategias de mercadeo y así lograr contribuir con el desarrollo económico regional.

Por lo anterior, el estudio se realizó con un enfoque cuantitativo, de tipo exploratorio y descriptivo; la muestra utilizada fue de 261 C.I seleccionadas de forma aleatoria, con un margen de error de $4 \%$ y un nivel de confianza de $94 \%$; en tal sentido, se diseñó el formulario compuesto por 45 preguntas, entregados a los Gerentes o representantes en cada C.I y una entrevista estructurada cerrada, aplicada a un funcionario de PROCOLOMBIA, los instrumentos fueron construidos a partir del diseño de seis perfiles (gerencia, producto, precio, plaza, promoción y tecnología - TIC's).

Método

\section{Tipo de estudio}

El tipo de estudio utilizado es no experimental, con un diseño de investigación descriptivo y transversal, bajo un enfoque positivista. Las variables estudiadas hacen referencia a la gerencia, la mezcla de mercadeo (producto, precio, plaza y promoción), la tecnología de información y comunicaciones, en relación con los objetivos específicos planteados en el estudio.

\section{Participantes}

El diseño de la muestra implementado en la investigación es probabilístico, aleatorio simple para población finita tomado de la base de datos emitida por la Cámara de Comercio de Cúcuta, en la cual se listan las C.I legalmente constituidas y con renovación del registro mercantil para el año 2010, en consecuencia, el tamaño de la muestra equivale a $n=126$ C.I ubicadas en la ciudad de San José de Cúcuta, en el departamento de Norte de Santander; estas empresas se caracterizan deben en esencia fomentar las exportaciones de productos de origen en nacional y posteriormente identificar y seleccionar nuevos nichos de mercado para comercializar los productos en un contexto global. Lo anterior corresponde con las políticas de incentivos las exportaciones realizado por el Gobierno de Colombia, para que de esta manera los productores locales, regionales y nacionales, concentren su actividad a la producción, mientras que la comercialización se delegue de forma estratégica a las organizaciones objeto de estudio. Otra característica demográfica de las C.I estudiadas, es que son microempresas, entre 2 y 4 empleados, con un alto grado de control de las operaciones comerciales y administrativas por parte del Departamento de Impuestos y Aduanas Nacionales (DIAN).

\section{Materiales e instrumentos}

Entre los materiales utilizados en la investigación, la recopilación de los datos se obtuvo a partir de dos instrumentos, en el modelo de encuesta, el primero, un formulario compuesto por cuarenta y cinco (45) preguntas entre demográficas, dicotómicas abiertas y cerradas, incluyendo el método de evaluaciones sumarias o escala de Likert, para especificar el nivel de acuerdo o desacuerdo de los encuestados a partir la una declaración en cada pregunta; dicho formulario se diseñó a partir de un cuadro de estructuración y sistematización de las variables del estudio. En este sentido, el segundo instrumento utilizado, corresponde a una 
entrevista estructurada y cerrada, constituida por doce (12) preguntas, aplicada a una funcionaria con cargo directivo de la Institución PROCOLOMBIA.

\section{Encuesta}

El análisis de las variables, se realizó a partir de la elaboración de la estructura diseñada por las dimensiones según el perfil de la gestión del proceso administrativo, el cual se define como el proceso de planificar, organizar, dirigir, y controlar actividades de los miembros de la organización y el empleo de todos los demás recursos organizacionales, con el propósito de alcanzar las metas establecidas por la organización (Stoner, Freeman y Gilbert, 1996) y las funciones de la mezcla de mercadeo, descritas como las cuatro variables que una compañía puede controlar para satisfacer las necesidades de un grupo objetivo del mercado: producto, plaza, precio y promoción, según (McCarthy, 1964) y tecnología de información y comunicaciones, como se establece en la tabla 1 .

Tabla 1.Estructura de dimensión de las variables de gestión administrativa de mercadeo de las C.I

ESTRUCTURA DE DIMENSION DE LAS VARIABLES DE GESTIÓN ADMINISTRATIVA DE MERCADEO DELAC.IDE CÚCUTA

\begin{tabular}{|c|c|c|c|}
\hline VARIABLE & $\begin{array}{l}\text { INSTRUMENTO } \\
\text { DE INVESTIGACIÓN }\end{array}$ & DIMENSIÓN & $\begin{array}{l}\text { PERFIL DE GESTIÓN } \\
\text { ADMINISTRATIVA }\end{array}$ \\
\hline GERENCIA & $\begin{array}{l}\text { Instrumento de } \\
\text { investigación } 1 . \\
\text { Sociedades de } \\
\text { Comercialización } \\
\text { Internacional(C.I) }\end{array}$ & Gestión Administrativa & $\begin{array}{l}\text { - P l a n e a c i ó } \\
\text { estratégica } \\
\text { - E s t } \mathrm{r} \text { u c t u r a } \\
\text { organizacional } \\
\text { - M o d e } 1 \text { o d e } \\
\text { Comunicación } \\
\text { - Filoso fía de la } \\
\text { gerencia } \\
\text { - Actividad económica } \\
\text { - Ta m a ñ o d e 1 a } \\
\text { empresa } \\
\text { - Gestión de marketing } \\
\text { - Talento Humano }\end{array}$ \\
\hline PRODUCTO & $\begin{array}{l}\text { Instrumento de } \\
\text { investigación } 1 \\
\text { Sociedades de } \\
\text { Comercialización } \\
\text { Internacional(C.I) }\end{array}$ & $\begin{array}{l}\text { Gestión de Marketing } \\
\text { Estratégico }\end{array}$ & $\begin{array}{l}\text { - Producto } \\
\text { - Marca } \\
\text { - Servicio } \\
\text { - Ventas }\end{array}$ \\
\hline
\end{tabular}


P R O M O C I Ó N Ninstrumento de Gestión de Marketing - T é c n i c a s d e /COMUNICACIÓ investigación 1. Estratégico Promoción

S o c i e d a d e s d e

- Publicidad

Comercialización

Internacional(C.I)

PRECIO

In s trume n to de investigación 1. Estratégico

- Establecimiento del Socied a d e s d e Comercialización Internacional (C.I)

PLAZA/DISTRIBUCI I n s t r u m e n t o d e ÓN investigación 1. Estratégico

S o c i e d a d e s d e

Comercialización

Internacional(C.I)

TECNOLOGÍA (TIC's) In s t r u m e n t o d e Software C.IINSTRUMENTO 1 in ve stiga ción 1 . So c i e d a d e s de Comercialización Internacional(C.I)
Hardware

Internet
TECNOLOGÍA (TIC's) I n s t r u m e n t o d e Fomento P R O E X P O R T investigación 2 . E $n$ t $r$ e $v$ i s t a PROEXPORT precio

- Mercado

- S e gmentos de 1 mercado

- Target

- Trans ferencia electrónica

- Pago electrónico

$\begin{array}{llllllll}-\mathrm{S} & \mathrm{o} & \mathrm{f} & \mathrm{t} & \mathrm{w} & \mathrm{a} & \mathrm{r} & \mathrm{e}\end{array}$ especializado

- Internet

- Intranet

- Extranet

- Hardware (Equipos)

- Sitio web comercial (Tienda virtual)

- E-busines

- Comercio electrónico

- E-marketing

- Comercio electrónico

- E-business

- E-marketing

- Investigación de mercados 


\begin{tabular}{|c|c|c|c|}
\hline VARIABLE & $\begin{array}{l}\text { INSTRUMENTO } \\
\text { DE INVESTIGACIÓN }\end{array}$ & DIMENSIÓN & $\begin{array}{l}\text { PERFIL DE GESTIÓN } \\
\text { ADMINISTRATIVA }\end{array}$ \\
\hline & & $\begin{array}{l}\text { I n t e } \mathrm{r} \text { a c c i ó } n \\
\text { Proexport/C.I }\end{array}$ & $\begin{array}{l}\text { - R e } 1 \text { a c i ó n y } \\
\text { c o m u n i c a c i ó n } \\
\text { Proexport/C.I }\end{array}$ \\
\hline & & Capacitación & $\begin{array}{l}\text { - Comercio electrónico } \\
\text { - E-business } \\
\text { - E-marketing } \\
\text { - Investigación de } \\
\text { mercados } \\
\text { - Comercio electrónico } \\
\text { - E-business } \\
\text { - E-marketing } \\
\text { - Investigación de }\end{array}$ \\
\hline
\end{tabular}

Fuente: Elaboración propia.

\section{Observación natural.}

A través de visitas de campo, se realizó el reconocimiento de las instalaciones de las C.I ubicadas en la ciudad de San José de Cúcuta, con el fin de conocer la infraestructura en la cual se desarrolla la actividades comercial de las empresas objeto del estudio; de esta manera el investigador tuvo la oportunidad de interactuar de manera informal con los directivos y gerentes encargados de la administración de cada negocio, y permitió establecer las rutas para la aplicación del instrumento dirigido a las empresas encuestadas.

\section{Procedimiento}

El estudio logró identificar las características de las Sociedades de Comercialización Internacional, de Cúcuta Norte de Santander, en relación con sus operaciones comerciales frente al uso y aplicación de estrategias de mercadeo, definidas como planes que especifican el impacto que una compañía espera alcanzar en cuanto a la demanda de un producto o una línea de producto dentro de un determinado mercado objetivo (Guiltinan, Paul y Madden, 2001); soportadas en plataformas tecnológicas, internet y la web 3.0, así como también, actividades de EMarketing y la utilización del modelo de comercio electrónico, el cual reúne a vendedores y compradores en un espacio virtual, de forma que ya no hay encuentro en las tiendas ni en las vitrinas. Las páginas de amazon.com y mercadolibre.com son pruebas de la rentabilidad de este modelo de negocios, en ese sentido, mediante el diseño y aplicación de la entrevista dirigida a un funcionario de PROCOLOMBIA, fue posible analizar el tipo de formación, apoyo logístico y asesorías técnicas que presta la Institución a las C.I para fomentar los conocimientos y el uso de internet y aplicaciones de mercadeo digital. 


\section{Resultados}

Según los resultados obtenidos para el perfil gerencia, el 50,98\% de las C.I encuestadas la misión está definida con una orientación hacia la exportación e importación de productos y servicios, por el contrario, cabe notar, que la visión empresarial de estas organizaciones es mínima, desde el punto de vista formal; lo cual significa la ausencia de un proceso de planeación estructurado y formalmente constituido, situación que ratifica la escasa gestión administrativa por parte de los empresarios del sector, lo cual, es congruente con el problema planteado; dado que, la actual crisis económica del sector afecta de manera directa la productividad de la región. Tomando en cuenta que tradicionalmente las ventas se han venido realizando al mercado de Venezuela y que en este momento el intercambio comercial entre los dos países se encuentra en una disminución acelerada, los empresarios no están preparados para dirigir sus esfuerzos comerciales hacia nuevos nichos de mercado en distinta posición geográfica, como se muestra en el gráfico 1.

\section{Gráfico 1. Perfil gerencia.}

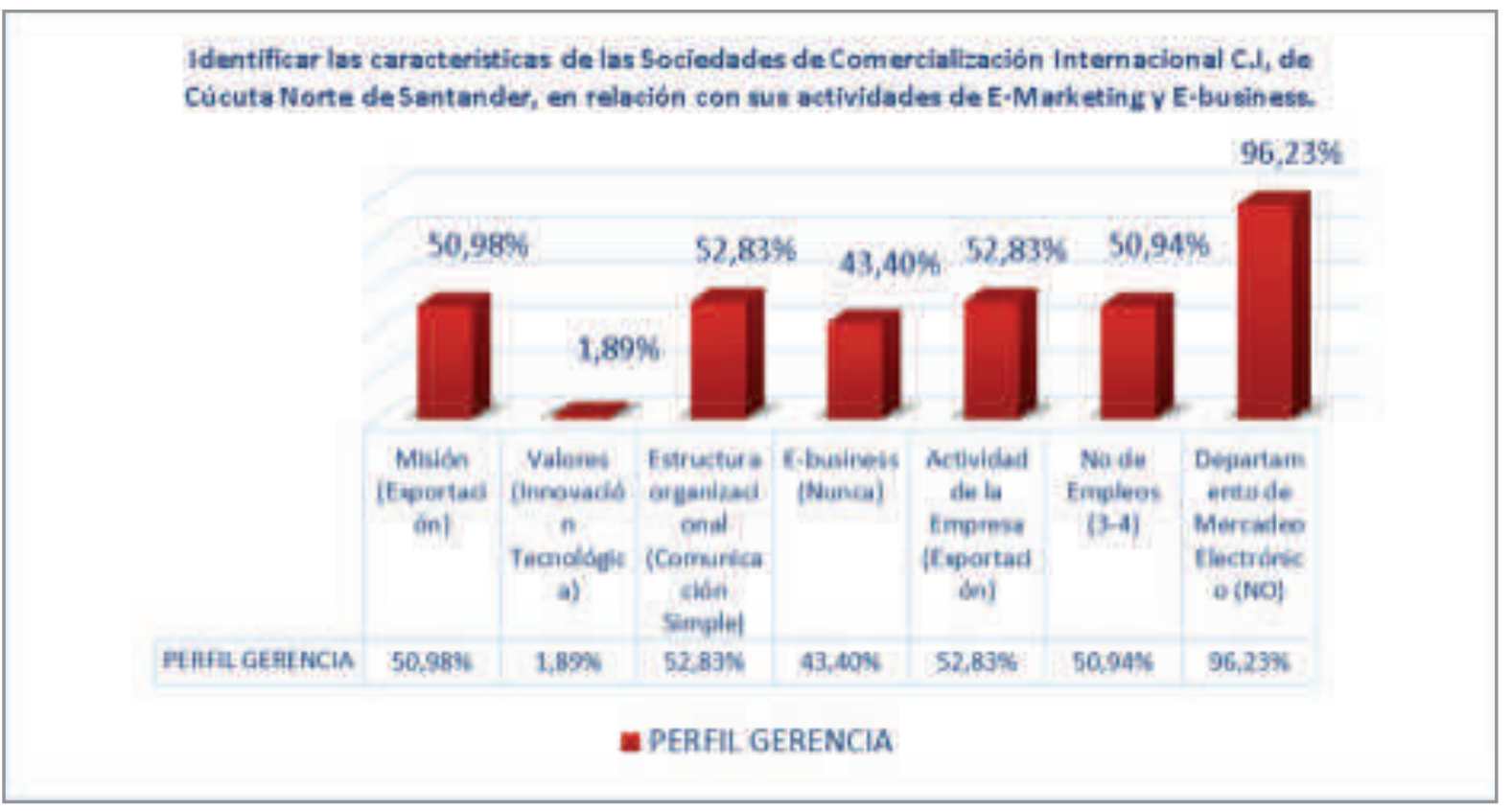

Fuente: Elaboración propia.

En ese sentido, se preguntó a las C.I estudiadas por la actividad económica de la empresa, el $52,83 \%$ contestó exportación, el 35,85\% realizan actividades de importación y exportación.

Los resultados obtenidos en el perfil Producto, revelan que el 79,25\% de bienes comercializados por las C.I corresponden a diferentes marcas, la operación comercial carece de una especialización de categorías, siendo los textiles y confección los más dinámicos con un $24,53 \%$, el 15,09\% manifiestan realizar actividades de postventa a través de correo electrónico, aunque el nivel de ventas a través de internet para el $75,47 \%$ es mínimo. 


\section{Gráfico 2. Perfil producto.}

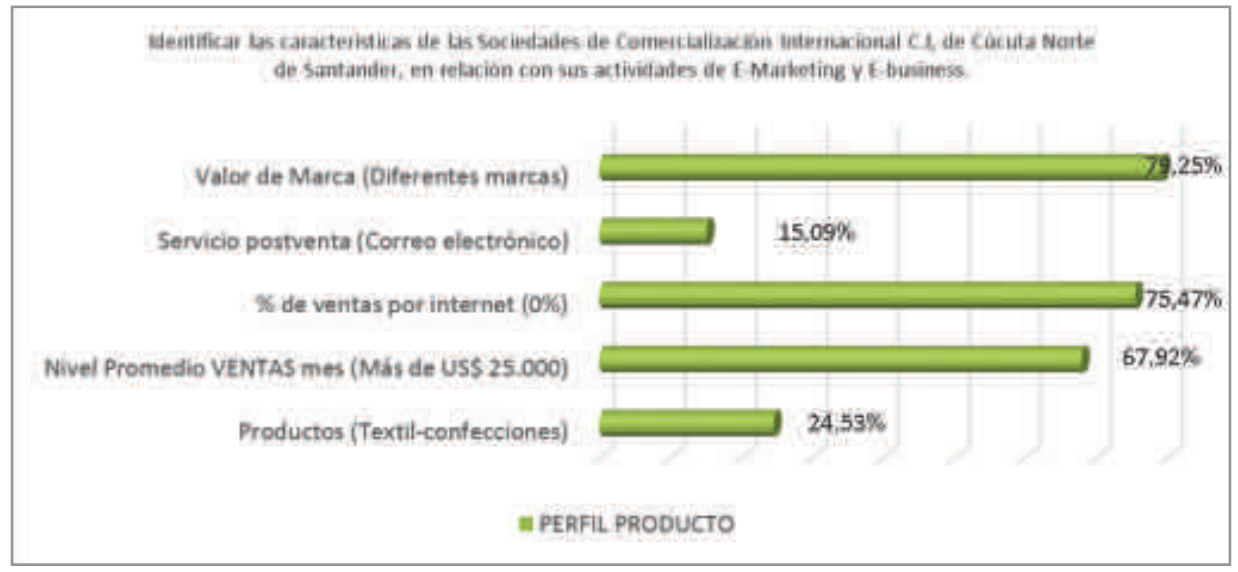

Fuente: Elaboración propia

Se identificó una alta dispersión en los productos comercializados hacia Venezuela por parte de las C.I, siendo los de mayor participación los textiles y confección con el 24,53\%, alimentos el $15,09 \%$, la arcilla el 9,43\%, minería, maquinaria y equipos y tecnología con un 5,66\%.

En lo referente al perfil promoción, el 15,09\% de las C.I realizan algún tipo de inversión publicitaria a través de medios digitales que no sólo transforma radicalmente conceptos clásicos como el de campaña o formato publicitario, sino que transforma significativamente el propio rol del receptor de los mensajes publicitarios (Martí, 2011), sin embargo, el 73,58\% de las empresas nunca han establecido algún tipo de técnica de promoción mediante medios digitales, lo cual se puede confirmar dado que el 73,58\% de los encuestados manifiesta que nunca han implementado descuentos en los productos a través de medios digitales.

Gráfico 3. Perfil promoción.

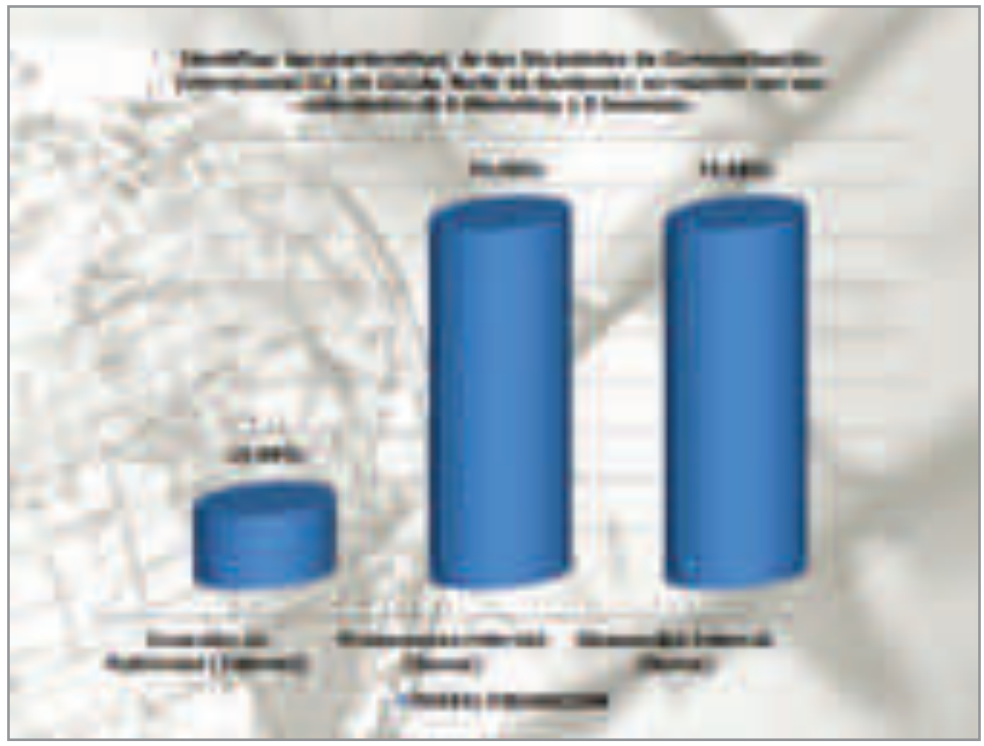

Fuente: Elaboración propia 
En el gráfico 4, se puede observar los hallazgos encontrados en el perfil plaza y precio, los resultados más representativos, hacen referencia a la técnica mediante la cual las empresas establecen los precios de venta, en ese sentido, el $41,51 \%$ lo hacen a partir de comparaciones de precios de la competencia en el mercado; así también, la logística de distribución se realiza desde Cúcuta hacia Venezuela, ya que el 83,02\% de las ventas realizadas son distribuidas en Venezuela.

\section{Gráfico 4. Perfil precio y plaza.}

\section{Identificar las cancteristicas de las Sociedades de Comercializacion Intemacional CI, de Cicuta Norte de Santandet, en relación con sus actividades de E-Marketing y E-business.}

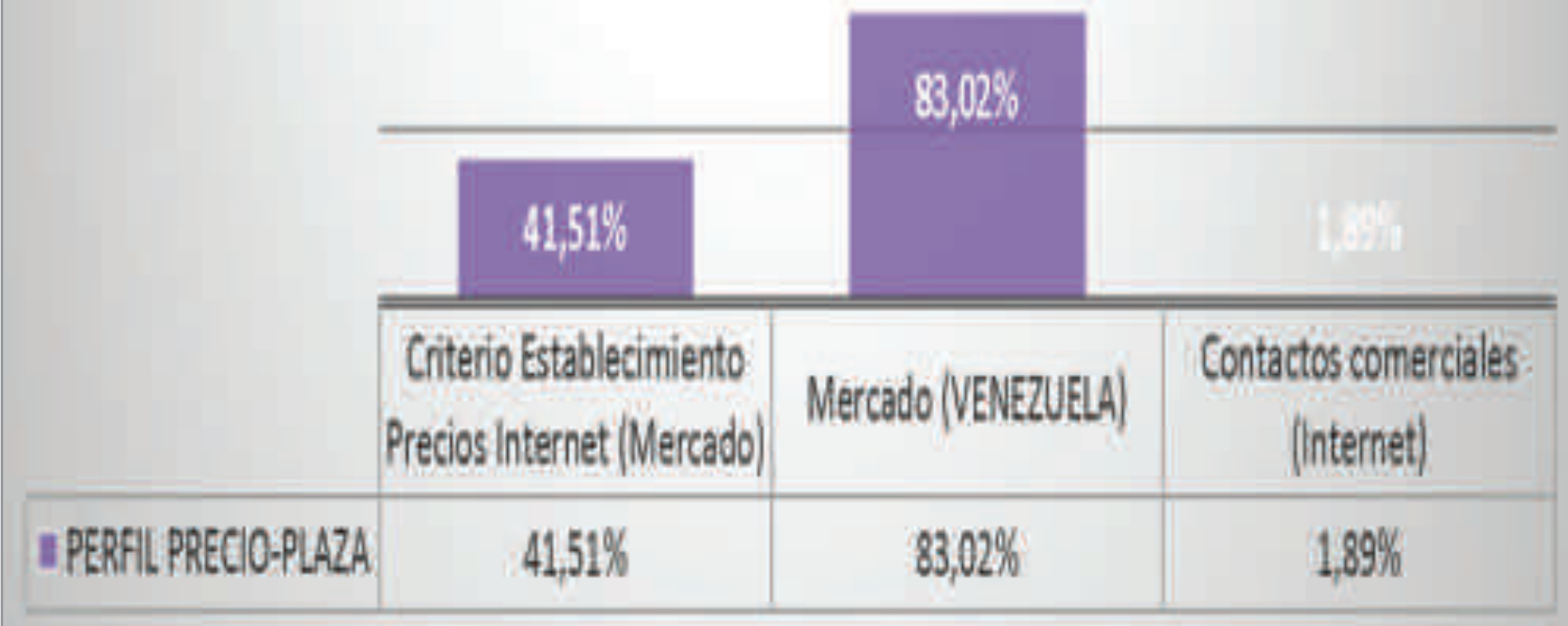

Fuente: Elaboración propia.

El perfil tecnológico TIC's, asociado al uso de los medios digitales por parte de las C.I en las operaciones comerciales y su relación con los clientes, muestra los siguientes resultados, para el $20,75 \%$ de la muestra las transacciones digitales son nada seguras, mientras que el $37,74 \%$ opinan que son seguras, lo cual permite establecer que existe interés en los empresarios en acercarse a los medios digitales, aunque es alto el nivel de desconfianza, es cierto que también se percibe que este tipo de transacciones pueden ser seguras. El 73,58\% opina no haber realizado ningún tipo de pago electrónico, relacionado con compras o ventas en internet, por el desconocimiento de la tecnología, desconfianza en la seguridad electrónica de datos y prefieren usar los medios tradicionales para tal fin. 
Gráfico 5. Perfil Tecnología de información y comunicación.

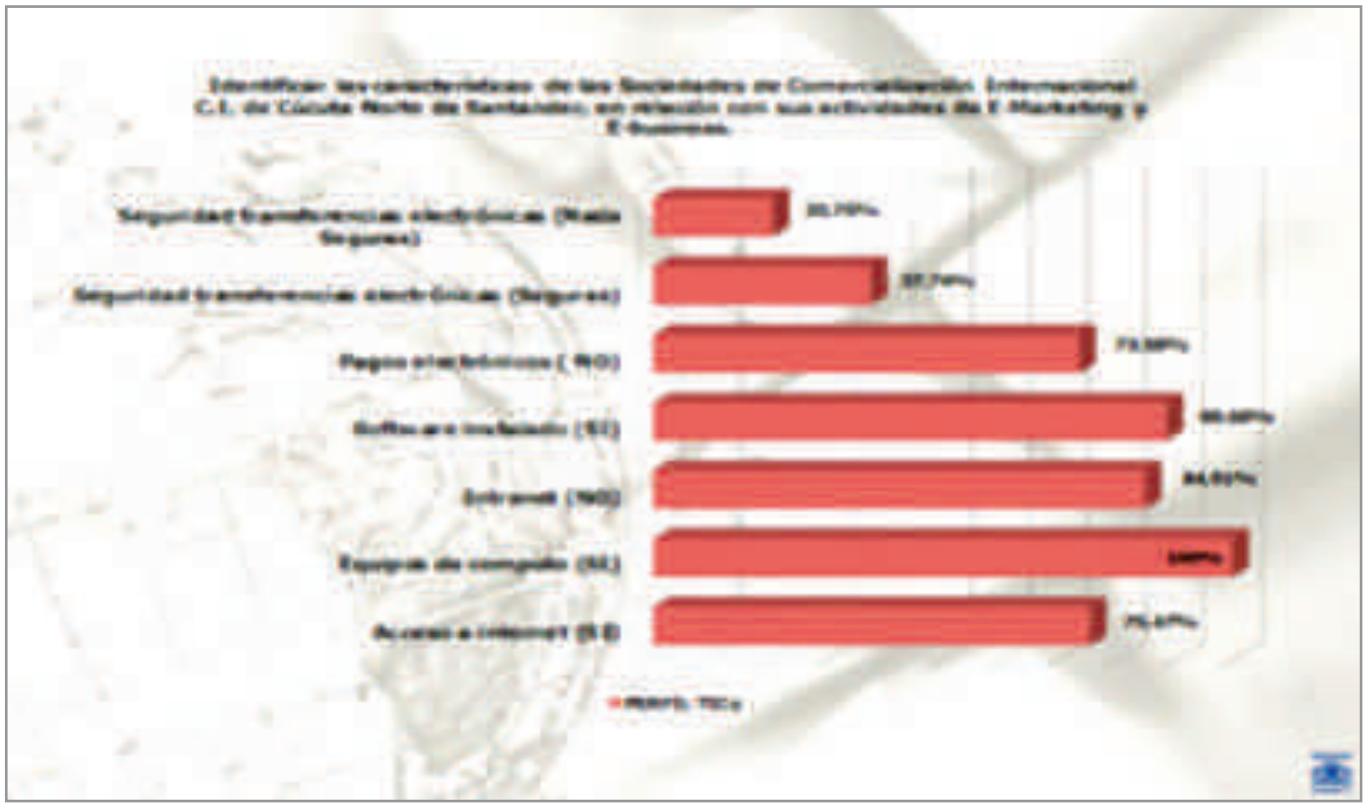

Fuente: Elaboración propia

El gráfico 6 expone otras características y resultados referentes al perfil TIC's; se preguntó a los encuestados si la empresa cuenta con alguna participación en el mercado virtual a través de una tienda virtual o página web comercial, para el $88,68 \%$ la respuesta fue negativa, el restante de la muestra tienen desconocimiento de estos conceptos del modelo de negocios en el mercado virtual. Para el $81,13 \%$ opinan y reconocen la importancia de mantener algún tipo de contacto con los clientes a través de los medios electrónicos ya que facilita la comunicación directa con el mercado objetivo.

\section{Gráfico 6. Perfil Tecnología de información y comunicación.}

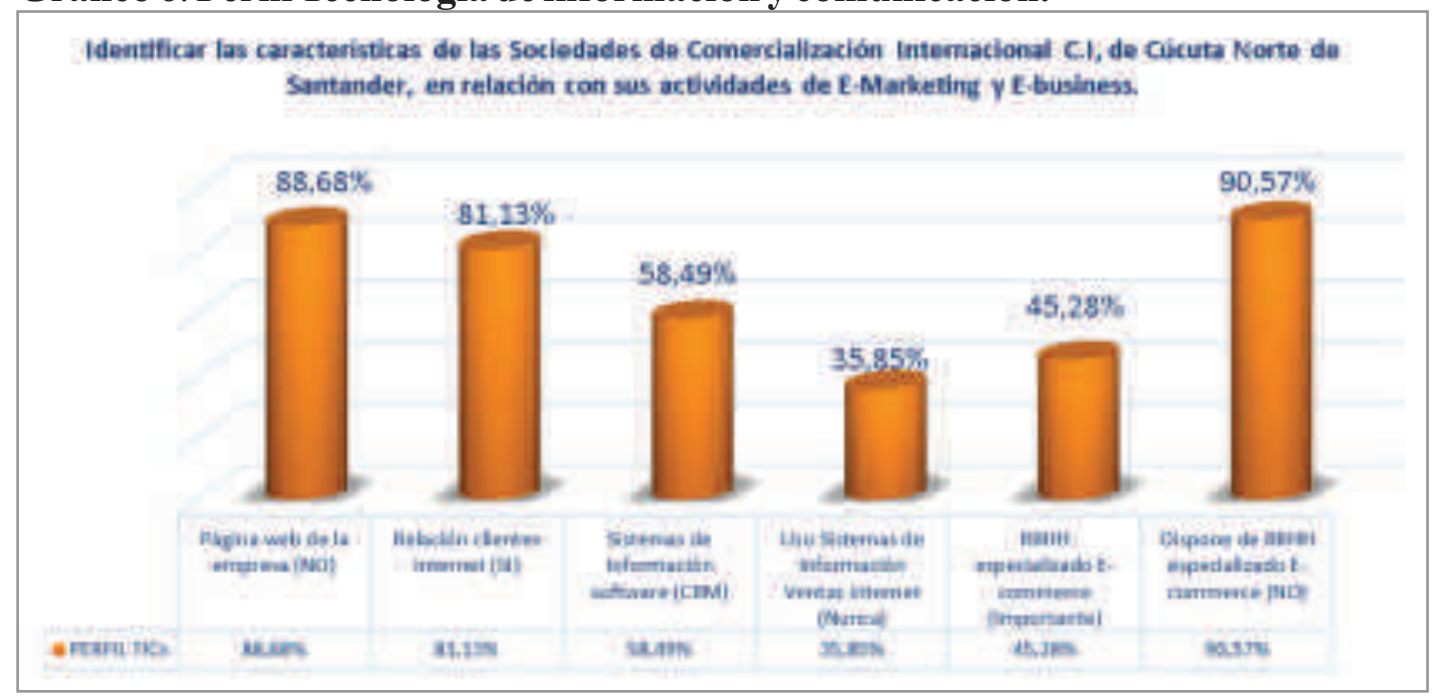

Fuente: Elaboración propia 
Por otra parte, para analizar la relación de PROCOLOMBIA en los procesos de formación, asesoría y capacitación en la implementación de las TIC aplicadas por las empresas objeto del estudio, los resultados fueron contemplados dentro del perfil TIC's; y para el 92,45\% de las C.I es necesario fomentar el comercio electrónico y la comunicación entre la Institución y las C.I para el 79,25\% es mínima.

\section{Comentarios}

El análisis realizado, a través de las variables estudiadas, determinó que efectivamente este tipo de organizaciones mantiene la operación del negocio mediante prácticas administrativas y de mercadeo netamente empíricas, sin contemplar los principios fundamentales de planeación, organización, dirección y control, la gestión se enfoca básicamente en la logística de transporte de la mercancía vendida, la elaboración y presentación de los documentos legales exigidos por el Departamento de Impuestos y Aduanas Nacionales (DIAN), de otra parte, es inexistente la filosofía de marketing en la gestión y proyección comercial, situación que agudiza la crisis en la que se encuentra el sector y la región, frente a indicadores como productividad y rentabilidad, que además acelera los descensos en los índices de empleo de la ciudad; Es preocupante, observar que durante el desarrollo de la investigación, la aguda crisis afectó directamente el proceso de recolección de la información en el trabajo de campo, ya que se presentó un cierre masivo y gradual de estas empresas, por lo cual se identificó gran inestabilidad, dadas las circunstancias.
En consecuencia, se puede concluir que se debe vincular más Instituciones públicas y privadas para fomentar el comercio internacional, como PROCOLOMBIA, la Cámara de Comercio de Cúcuta, Fenalco, etc. Mediante el diseño de programas de capacitación y asesoría empresarial y temas relacionados con marketing y medios digitales, así como también, en E-business el cual se refiere a todo el hardware y software y conocimiento sobre computadores que ofrecen una plataforma para que los negocios (Wells, Moriarti y Burnett, 2007).

Por lo anterior, es fundamental preparar a los empresarios en el uso de estas técnicas de intercambio a través de internet y la web 3.0, que actualmente no encuentran este apoyo y el uso de los medios de pago electrónico los cuales "se ha catalogado como una forma de extinguir las obligaciones. Consiste en la satisfacción, ejecución o cumplimiento de la presentación debida por el deudor que puede ser de dar, hacer o no hacer" (Rojas, 2013).

\section{Agradecimientos}

Agradezco en primera instancia a Dios, por concederme salud y vitalidad para culminar exitosamente esta meta propuesta, a mi familia, mi madre Flor de María Elena Díaz de Andrade y mi padre Hernando Andrade Barrios, por su apoyo incondicional; a mis hijos, Mariana y Diego Alejandro por el tiempo que deje de dedicarles, por su comprensión y paciencia; un agradecimiento especial a los empresarios directivos de la Sociedades de Comercialización Internacional C.I de Cúcuta Norte de Santander, por la amable contribución al desarrollo de la 
presente investigación, mediante la dedicación del tiempo necesario para resolver cada cuestionario y demás personas que contribuyeron al desarrollo de la investigación.

\section{Referencias}

Hernández , A., \& Küster, I. (2012). Brand impact on purchasing intention. An approach in virtual social. 1(2).

McCarthy, E. J. (1964). Basic Marketing. A Managerial Approach. Homewood, IL: Richard D. Irwin.

British Broadcasting Corporation. (16 de febrero de 2005). Corporación Británica de Radiodifusión (BBC- MUNDO). Recuperado el

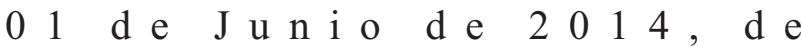
http://news.bbc.co.uk/hi/spanish/business/news id_4269000/4269295.stm

Burk Wood, M., \& Gómez Borja, M. (2004). El plan de marketing: guía de referencia. Editorial Alhambra S. A.

Cruz Herradón, , A. (2009). Marketing electrónico para PYMES, cómo vender, promocionar y posicionarse en internet. . México: RA-MAEditorial.

Departamento Nacional de Planeación. (2007). Agenda Interna para la Productividad y la Competitividad. Bogotá: DNP-Agenda Interna.

Duarte Cueva, F. (2010). Economía digital, sitios web y PYMES del sector artesanías en el
Perú. vol. 5( núm. 9).

Flórez Rojas, M. L. (2013). La reversión del pago dentro del contexto del comercio electrónico y sus implicaciones prácticas en el régimen jurídico colombiano.(10).

Guiltinan, J., Madden, T., \& Paul, G. (2001). Gerencia de Marketing: estrategias y programas. Bogotá: McGraw-Hill.

Hernández, M. C. (2014). ¿Qué le espera al comercio colombo-venezolano en el 2014? Portafolio.

Kotler, P., \& Armstrong, G. (2008). Fundamentos de Marketing. México: Prentice Hall.

Martí Parreño, J. (2011). Marketing y publicidad en internet. Bogotá: Ediciones de la U.

Rayport , J., \& Jaworski , B. (2003). ECommerce. México: Mcgraw-Hill Interamericana de México.

Schiffman, L., \& Kanuk, L. (2005). Comportamiento del consumidor. México: Pearson Education .

Stanton, W., Etzel, M., \& Walker, B. (2004). Fundamentos de marketing. México, D.F: McGraw-Hill Interamericana.

Stoner, J., Gilbert, D., \& Freeman, E. (1996). Administración. México: Prentice Hall Hispanoamericana S.A. 
Wells, W., Burnett, J., \& Moriarty, S. (2007). Publicidad: principios y práctica. Pearson Educación.

Zysman, J., \& Weber, S. (01 de 08 de $2001)$. EconPapers- Escritos
Económicos. (EconPapers) Recuperado el 15 de $\begin{array}{llllll}\mathrm{M} \text { a y o } & \mathrm{d} \text { e } & 2014, & \mathrm{~d} \mathrm{e}\end{array}$ http://econpapers.repec.org/paper/cdlucbrie/qt7 zx3g1qn.htm 